\title{
Mn Katkılı PIN-PMN-PT Tekil Kristalinin Dielektrik Katsayısı, Relaksiyon Zamanı ve Aktivasyon Enerjisinin Hesaplanması
}

\author{
Sinan AKSOY ${ }^{1 *}$, Arzu KURT ${ }^{2}$ \\ ${ }^{1}$ Mithatpaşa Ortaokulu, Tekirdağ, Türkiye. \\ ${ }^{2}$ Çanakkale Onsekiz Mart Üniversitesi, Uygulamalı Bilimler Fakültesi, Enerji Yönetimi Bölümü, Çanakkale, Türkiye. \\ Sorumlu yazar e-posta: ${ }^{1}$ snnaksy@gmail.com ORCID ID: https://orcid.org/0000-0002-5537-2966 \\ e-posta: ${ }^{2}$ arzukurt@comu.edu.tr ORCID ID: https://orcid.org/0000-0003-3196-100X \\ Geliş Tarihi: 01.07.2020 Kabul Tarihi: 13.02.2021
}

Öz

\begin{abstract}
Relaksör-ferroelektrik malzemeler 1950 li yıllarda keşfedilmiştir, birçok uygulamada kullanılmasına rağmen hala bazı mekanik ve elektrik özellikleri tam olarak anlaşılamamıştır. Özellikle ferroelektrik faz geçişlerindeki, mekanik ve elektriksel davranımlarının sıcaklık bağımlılıklarını tam olarak modelleyebilen bir yaklaşım eksikliği vardır. Bu çalışmada, Landau fenomonolojik teori kullanılarak, Mn katkılı PIN-PMNPT tek kristal karışım malzemenin ferroelektrik rhombohedral-monoklinik faz geçişinde $\left(T_{C}=112{ }^{\circ} \mathrm{C}\right) 512$ ve $584 \mathrm{~cm}^{-1}$ Raman modları için; kendiliğinden polarizasyon, dielektrik duyarlık ve gevşeme zamanı sıcaklığa bağlı olarak hesaplanmıştır. Landau fenomonolojik teoriden türetilen dielektrik duyarlık bağıntısı, deneysel veriler ile uyumlu sonuçlar vermiştir. Ayrıca, ferroelektrik rhombohedral fazda farklı sıcaklık aralıkları için aktivasyon enerjileri pseuode-spin fonon ve enerji dalgalanma model yaklaşımları kullanılarak hesaplanmıştır. Her iki model kullanılarak hesaplanan sönümleme sabiti değerlerimiz, gözlemlenen verilerle uyumlu çıkmıştır.
\end{abstract}

Anahtar kelimeler Relaksör-ferroelektrik; Raman mod; Landau model; Faz geçişi; Aktivaston enerjisi

\section{Calculation of Dielectric Coefficient, Relaxation Time and Activation Energy of Mn Compounded PIN-PMN-PT Mono Crystal}

\begin{abstract}
Relaxor-ferroelectric materials were discovered in the 1950s, and although they are used in many applications, some of their mechanical and electric properties have not fully understood yet. There haven't been an adequate approach that can precisely model the temperature dependencies of their mechanical and electrical behaviour, especially in ferroelectric phase transitions yet. In this study, using Landau phenomenological theory, Mn-doped PIN-PMN-PT single crystal mixture material ferroelectric rhombohedral-monoclinic phase transition $\left(\mathrm{TC}=112{ }^{\circ} \mathrm{C}\right.$ ) for 512 and $584 \mathrm{~cm}^{-1}$ Raman modes; spontaneous polarization, dielectric sensitivity and relaxation time were calculated based on temperature. The dielectric precision correlation derived from Landau phenomenological theory yielded results consistent with experimental data. Furthermore, activation energies for different temperature ranges in the ferroelectric rhombohedral phase were calculated using pseudospin Phonon and energy fluctuation model approaches. Our damping constant values, which were calculated using both models, were good well consistent with the observed data.
\end{abstract}

(C) Afyon Kocatepe Üniversitesi

\section{Giriş}

Piezoelekrik malzemeler, mekanik enerjiyi elektrik enerjisine dönüştürme özelliğine sahiptirler. Mekanik enerjiyi elektrik enerjisine çevirebilen bu özel malzemelerin tıp, ultrason ve telekomünikasyon gibi pek çok alanda uygulamaları bulunmaktadır. Relaksör-ferroelektrik malzemeler, bir dış elektrik alanı altında şekillerini yüksek oranda değiştiren ferroelektrik özellikli malzemelerdir. Relaksör-ferroelektrik özellik gösteren malzemelerin keşfinin üzerinden 65 yıl geçmiş olmasına rağmen, bu etkinin mekanizması hala tam olarak anlaşılamamıştır. Bu yüzden, relaksör- 
ferroelekrik malzemeler yoğun madde fiziğinin en çok araştırılan konularından birisi olmuştur.

PMN olarak da bilinen $\mathrm{Pb}\left(\mathrm{Mg}_{1 / 3} \mathrm{Nb}_{2 / 3}\right) \mathrm{O}_{3}$ gibi klasik relaksör-ferroelektrik malzemeler, yönelim ve yük düzensizlikleri ile karakterize edilebilen katı perovskit çözeltilerdir (Cross 1987, Cross 1993). Soğutulmaları sırasında simetrilerinde bir değişim gözlenmeyen bu çözeltilerin, dielektrik ve elektromekanik tepkilerinde, uygulanan elektrik potansiyel frekansına bağıı ani değişimler gözlenmektedir (Cross 1987, Cross 1993, Kutnjak et al. 1996). Relaksör-ferroelektrik malzemeler dipolar özelliğe sahip olmasına rağmen, ferroelektrik faz geçişleri yüksek elektrik alan uygulanması ile gözlemlenebilmektedir (Böttger et al. 1992, Colla et al. 1995, Westphal et al. 1992), ayrıca küçük ve rastgele sıralanmış polar nano-bölgelerden oluşması onları elektrik alan ile daha etkin bir şekilde etkileşime geçmesine sebep olmaktadır (Blinc et al. 1999, Burns and Dacol 1983, Kutnjak et al. 1996, Pirc et al. 2002).

PMN-PT temel karışım sistemi sıcaklık ve elektrik alan büyüklüğüne bağlı olarak farklı faz geçişleri gösterebilmektedir. Saf PMN elektrik alan etkisiyle ferroelektrik faza ek olarak yüksek sıcaklıkta ergodik relaxör, düşük sıcaklıkta ise ergodik olmayan relaxör faza sahiptir. Yüksek PT konsantrasyonlarında, düşük sıcaklıklarda ferroelektrik tetragonal $(T)$, rombohedral (R), monokilinik (M) ve ortohorhombik (O), yüksek sıcaklıklarda ise paraelektrik kübik (C) fazlar gözlenmektedir. Kendiliğinden polarizasyon yönelimleri tetragonal (P4mm) fazda [001], rombohedral (R3m) fazda [111] ve ortohorhombik (mm2) fazda [110] olmaktadır. Rombohedral ve tetragaonal fazlar arasında olası üç monoklinik faz (MA, MB ve MC) bulunmaktadır (Davis et al. 2005, Davis et al. 2006, Viehland and Powers 2001).

Zhao vd. (2007), farklı yönelimlerde [111], [110] ve [100] elektrik alan uygulayarak PMN için faz geçişlerini incelemişlerdir. Elektrik alanın [111] doğrultusunda uygulandığında kritik noktanın varlığını onaylamalarına rağmen, [110] ve [100] doğrultusunda $7.5 \mathrm{kV} / \mathrm{cm}$ elektrik alan kuvvetine kadar yapılan ölçümlerde kritik nokta gözlemlememişlerdir (Zhao et al. 2007). Buna karşılık, Kutnjak vd. (2006)'a göre, [110] doğrultusundaki PMN-PT sisteminde kritik noktaların varlığını belirlemişlerdir. Ayrıca dışelektrik alan etkisi altındaki PMN-PT sistemi, $P M N$ ye göre daha düşük elektrik alan altında faz geçişleri sergilemiştir. [110] ve [111] doğrultularında dış elektrik alan uygulandığında, PMN-PT sisteminin faz geçişleri incelenmiş, polarizasyon değişim mekanizması ve piezoelektrik tepkileri elektrik alansıcaklık faz diyagramındaki gözlemler ile ilişkilendirilmiştir (Kutnjak et al. 2006).

Son yıllarda, manganez (Mn) gibi katkı maddeleri ekleyerek relaksör-PT tek kristallerinin "sertleştirilmesi" için çok sayıda çaba sarf edilmiştir. Kurşun-magnezyum-niobat (PMN) ve kurşun titanatın (PT) katı çözeltilerine dayanan tek kristaller, bilinen en iyi kurşun-zirkonyumun katkılı PMN den çok daha fazla piezoelektrik, piroelektrik ve elektro-optik özellikleri birleştiren, işlevsellik bağlamında çok umut verici sistemler olarak ortaya çıkmıştır (Wang et al. 2014). Pratik uygulamalarda sıcaklık değişiminden kaynaklı piezoelektrik katsayısı, elektromekanik bağlaşma katsayısı ve mekanik kalite değişimi, elektromekanik cihazların performansını doğrudan etkileyecektir. Bu nedenle, sıcaklığın Mn katkılı üçlü PIN-PMN-PT tekil kristal özelliklerini nasıl etkilediğini anlamak çok önemlidir. Liu vd. (2019) Mn-0.15PIN-0.55PMN-0.30PT kristalinin dielektrik, piezoelektrik ve ferroelektrik özelliklerini içeren elektriksel özelliklerin sıcaklığa bağlılığını araştırmış ve sıcaklığa bağlı faz geçiş karakteristiklerini Raman spektroskopik yöntemle incelemiştir (Liu et al. 2019).

Bu çalışmada, Mn-0.15PIN-0.55PMN-0.30PT kristal sisteminin ferroelektrik-rhombohedral fazda sıcaklığa bağlı kendiliğinden polarizasyonu, Landau fenomonolojik modelden türetilen dielektrik ifadesinin deneysel verilere fit edilmesiyle elde edilen katsayılar kullanılarak hesaplanmıştır. Mn0.15PIN-0.55PMN-0.30PT'nin ferroelektrikrhombohedral fazdan ferroelektrik-monoklinik faza geçiş için incelenen Raman modlarının sönümleme sabitini ( $\Gamma$ - çizgi genişliği) hesaplamak için, psödospin-fonon bağlı (PS) modelini (Lahajnar et al. 1974) ve enerji dalgalanması (EF) modelini (Schaack and Winterfeldt 1977) kullandık. Bu hesaplamalarda, düzen parametresi olarak tanımladığımız kendiliğinden polarizasyonun belirlenen değerleri kullanılmıştır. Tüm bunlara ek 
olarak, her iki model için (PS ve EF model), Mn0.15PIN-0.55PMN-0.30PT'nin farklı Raman modlarında gevşeme zamanı ve aktivasyon enerjilerini sıcaklığın bir fonksiyonu olarak belirledik. Sönümleme sabiti için deneysel verilere fit ederek hesaplanmış olduğumuz değerler kullanılmıştır.

\section{Materyal ve Metot}

Klasik Landau fenomonolojik teorisi, ferroelektrik perovskit kristal ailesi malzemelerinde tetragonal, ortorombik, rhombohedral, monoklinik gibi ferroelektrik faz geçişlerinin açıklanmasında etkin bir yaklaşım vermektedir. Ayrıca, bu model ferroelektrik faz geçiş gösteren birçok perovskit türü kristalin faz geçişlerinin modellenmesinde etkin bir yaklaşım metodu sunmaktadır (Kim et al. 2018). Belirli sıcaklıklarda bulunan güçlü bağlanma etkisi altındaki tetragonal yapıların, ferroelektrik davranışları Landau fenomenolojik teorisi kullanılarak incelenebilir. Günümüzde LandauDevonshire serbest enerji fonksiyonları rombohedral fazdaki PIN-PMN-PT kristallerin dielektrik, piezoelektrik ve ferroelektrik özelliklerini incelemek için önerilmiş ve yapılan incelemelerde rombohedral fazdaki PMN-0.30PT ve PMN-0.36PT kristallerinin deneysel veriler ile oldukça uyumlu sonuçlar gösterdiği görülmüştür (Lu et al. 2020). Bu çalışmada, Mn-0.15PIN-0.55PMN-0.30PT temel karışım sisteminin ferroelektrik-rhombohedral ferroelektrik-monoklinik faz geçiş bölgesindeki karakteristiğini tanımlamak için yüksek dereceli Klasik Landau fenomenolojik modeli kullanılmıştır. Landau fenomenolojik modelde sistemin serbest enerjisi, düzen parametresinin seri açılımı ile oluşturulmaktadır. Sistem düzen parametresi $\mathrm{P}$; sistemdeki moleküllerin kendiliğinden sahip oldukları polarizasyondur. Molekülün elektrik alan altında sahip olduğu düzen parametresi kendiliğinden polarizasyonu üzerinden seri açılımı ile türetilen Landau fenomenolojik modelin ifadesi;

$$
F=a_{0}+a_{2} P^{2}+a_{4} P^{4}+a_{6} P^{6}-P E
$$

olarak tanımlanmıştır. İfadede, F sistemin serbest enerjisini, $\mathrm{P}$ sistemdeki moleküllerin sahip olduğu polarizasyonu, E normalize edilmiş dış elektrik alan kuvvetini, $a_{2}, a_{4}$ ve $a_{6}$ düzen parametrelerinin katsayılarını belirtmektedir. Bu modelde ikinci derece $\left(\mathrm{P}^{2}\right)$ düzen parametresi katsayısı sıcaklığa bağlı iken, dördüncü $\left(P^{4}\right)$ ve altıncı $\left(P^{6}\right)$ dereceli terimlerin katsayıları sıcaklıktan bağımsızdır. Ayrıca $a_{2}$ katsayısı sıcaklığın değişimi ile ilişkili $a_{2}=\alpha\left(T-T_{C}\right)$ olarak tanımlanmıştır, $\mathrm{T}$ ortam sıcaklığı, $T_{c}$ faz geçiş sıcaklığıdır. Sistem, $a_{4}<0$ durumunda birinci dereceden faz geçişi, $a_{4}>0$ durumunda ise ikinci dereceden faz geçişi göstermektedir, $a_{6}$ katsayısı ise her koşulda pozitif değere sahip olmaktadır. Termodinamik dengede, Mn-0.15PIN-0.55PMN-0.30PT karışım sisteminin serbest enerjisi $(F)$, ferroelektrik rhombohedralmonoklinik faz geçişinde minimum olur $\left(T<T_{C}\right)$. Serbest enerjinin polarizasyona göre minimizasyonundan $(\mathrm{dF} / \mathrm{dP}=0)$;

$\frac{d F}{d P}=2 a_{2} P+4 a_{4} P^{3}+6 a_{6} P^{5}-E$

$E=2 a_{2} P+4 a_{4} P^{3}+6 a_{6} P^{5}$

elde edilir. Dış elektrik alanın sıfır olduğu durumda $(\mathrm{E}=0)$;

$0=a_{2} P+2 a_{4} P^{3}+3 a_{6} P^{5}$

Denk.(4)' ü P parantezine aldığımızda;

$P \cdot\left(a_{2}+2 a_{4} P^{2}+3 a_{6} P^{4}\right)=0$

olur, bu denklemin çözümünde $\mathrm{P}=0$ durumu, sistemde tam düzensizliğe karşılık geldiğinden Denk.(5) in ikinci kısmının çözümü bulunmalıdır. Bunun için Denk.(5) in ikinci kısmı için $X=P^{2}$ dönüşümü yapıldığında;

$3 a_{6} X^{2}+2 a_{4} X+a_{2}=0$

Bu denklemin pozitif ve negatif kökleri;

$P^{2}=\frac{-a_{4}}{3 a_{6}} \pm \frac{1}{3 a_{6}} \sqrt{a_{4}^{2}-3 a_{2} a_{6}}$

$a_{4}<0$ ve $a_{6}>0$ durumunda $\mathrm{P}^{2}$ nin pozitif kökü elde edilir, pozitif kök düzenin olduğu ferroelektrik rhombohedral faza karşılık gelmektedir. 
Ferroelektrik özellik gösteren malzemelerde ters dielektrik duyarlılık sabitinin sıcaklığa bağlı değeri, Klasik Landau fenomenolojik teorisinde Serbest enerji bağıntısından türetilebilir;

$\chi^{-1}=\frac{\partial^{2} F}{\partial^{2} P}$

Yurtseven vd. (2017), $\frac{a_{2} a_{6}}{a_{4}^{2}}<<1$ yaklaşımını kullanarak, ferroelektrik düzenli fazda $(T<T)$ ters dielektrik duyarlılık sabitinin ve kendiliğinden polarizasyonun sıcaklığa bağlı denklemlerini türetmişlerdir (Yurtseven vd. 2017);

$$
\begin{aligned}
& \chi^{-1}=\left(\varepsilon_{R}-1\right)^{-1}=-12 \alpha\left(T-T_{C}\right)+\frac{16 a_{4}^{2}}{3 a_{6}} \\
& P^{2}=\alpha \frac{\left(T-T_{C}\right)}{2 a_{4}}-\frac{2 a_{4}}{3 a_{6}}
\end{aligned}
$$

$\mathrm{Bu}$ çalışmada, Mn-0.15PIN-0.55PMN-0.30PT relaksör ferroelektrik malzemenin rhombohedral fazdaki, dielektrik katsayısının deneysel ölçüm değerleri kullanılarak, $\alpha, a_{4}$ ve $a_{6}$ katsayıları belirlenmiştir (Liu et al. 2019).

Ising Psödospin-fonon model, moleküler kristallerde fononların sıcaklık bağımlılığını elde etmek için kullanılan modeldir. Yamada ve ark., 1972'de $\mathrm{NH}_{4} \mathrm{Br}$ için sadece bir psödospin ve bir fonon dikkate alınarak sönümleme sabitinin sıcaklığa bağıı̆ı̆ını araştırmak için birleştirilmiş bir model geliştirmişlerdir (Yamada et al. 1972). Matsushita (1976), birden fazla pseudospin ve birden fazla fonon etkileşimini dikkate alarak bu modeli genişletmiştir (Matsushita 1976). Sönümlenme sabitinin düzen parametresi ve sıcaklığa bağlı ifadeleri, kritik sıcaklık ( $\left.T_{C}\right)$ yakınında Psödospinfonon etkileşimleri dikkate alınarak Lahajnar tarafından PS model (Lahajnar et al. 1974), Schaack tarafından psödospin-fonon etkileşimi için başka bir yaklaşım kullanılarak EF model (Enerji dalgalanması) (Schaack and Winterfeldt 1977) türetilmiştir;

$$
\Gamma_{S P}=\Gamma_{0}+A\left(1-P^{2}\right) \ln \left(\frac{T_{C}}{T-T_{C}\left(1-P^{2}\right)}\right)
$$

$$
\Gamma_{P S}=\Gamma_{0}^{\prime}+A_{0}^{\prime}\left[\frac{T\left(1-P^{2}\right)}{T-T_{C}\left(1-P^{2}\right)}\right]^{1 / 2}
$$

Burada $P$ sistemin düzen parametresidir (kendiliğinden polarizasyon). Denklem (11) ve (12) 'de, $\Gamma_{0}\left(\Gamma_{0}^{\prime}\right)$ arka plan sönümleme sabitini (çizgi genişliği) ve $A_{0}\left(A_{0}^{\prime}\right)$ genliği temsil eder. Daha önce yapmış olduğumuz çalışmamızda, DMFeM $(\mathrm{M}=\mathrm{Ni}$, $\mathrm{Zn}, \mathrm{Cu}, \mathrm{Fe}$ ve $\mathrm{Mg}$ ) ferroelektrik MOF malzemenin, $\rho\left(\mathrm{NH}_{2}\right)$ modunun IR frekanslarına karşılık gelen FWHM değerleri, yukarıda tanımlanmış olan PS ve EF modeller kullanılarak çeşitli sıcak aralıkları için analizler yapılmıştır (Kurt vd. 2019).

Bu çalışmada, ayrıca PS ve EF model için düzen parametresinin sıcaklık bağımlılı̆ı kullanılarak, Mn$0.15 \mathrm{PIN}-0.55 \mathrm{PMN}-0.30 \mathrm{PT}$ temel karışımının $510 \mathrm{~cm}^{-}$ ${ }^{1}$ ve $584 \mathrm{~cm}^{-1}$ Raman modları için gevşeme zamanı $(\tau)$ sıcaklığa bağlı olarak hesaplanmıştır (Lahajnar et al. 1974).

$$
\tau=\frac{\Gamma}{P^{2}}
$$

Son olarak, her iki Raman modu için FWHM değerleri kullanılarak farklı sıcaklık aralıkları için aktivasyon enerjisi hesaplanmıştır. Bu hesaplamalarda, Hendrickson ve Bray yaklaşımı kullanılmıştır (Hendrickson and Bray 1973);

$$
\Gamma=\Gamma_{v i b}+C \cdot \exp \left(\frac{-U}{k_{B} T}\right)
$$

burada $\Gamma_{\text {vib }}$ titreşim frekansını, $\mathrm{k}_{\mathrm{B}}$ Boltzman sabitini ifade etmektedir, $\mathrm{C}$ ise sabit katsayıdır.

\section{Bulgular}

Bu çalışmada, Ferroelektrik faz geçiş sıcaklı̆̆ı $T_{C}$ 'nin $\left(T_{C}=112{ }^{\circ} \mathrm{C}\right)$ altındaki rhombohedral-monoklinik faz geçişi için Fenomonolojik Landau teorisini kullanarak, Mn-0.15PIN-0.55PMN-0.30PT kristalinin ters dielektrik duyarlılı̆ının, polarizasyonun (düzen parametresi) ve gevşeme zamanının sıcaklığa bağımlılığını ve farklı sıcaklık aralıklarında aktivasyon enerjisini belirledik. Bu hesaplamalar, [001] 
oryantasyonunda Mn-0.15PIN-0.55PMN-0.30PT kristalinin $510 \mathrm{~cm}^{-1}$ ve $584 \mathrm{~cm}^{-1}$ Raman modları için yapılmıştır. Literatürde, Raman modlarının genişlemesi, birleşmesi ve frekans kaymasının tipik olduğu ve perovskit gevşetici bazlı ferroelektrikler için ferroelektrik faz geçişleri ile ilişkili olduğu belirtilmektedir (Liu et al. 2016). Mn-0.15PIN$0.55 P M N-0.30 P T$ kristalinin $510 \mathrm{~cm}^{-1}$ ve $584 \mathrm{~cm}^{-1}$ Raman modları, kristal yapıdaki $\mathrm{Nb}-\mathrm{O}-\mathrm{Nb}$ gerilme modlarına karşılık gelmektedir (Svitelskiy et al. 2003). Mn-0.15PIN-0.55PMN-0.30PT ferroelektrik kristal malzemenin ferroelektrik-rhombohedral fazında, Liu vd. (2019) almış oldukları deneysel verileri Denk. 9'a fit yaparak, $\alpha, a_{4}$ ve $a_{6}$ katsayılarını belirlerdik (Çizelge 1) (Liu et al. 2019).

Çizelge 1. Mn-0.15PIN-0.55PMN-0.30PT tekil kristal ferroelektrik-rhombohedral faz $(\mathrm{T}<\mathrm{TC})$ için Denk. (9)'a göre hesaplanan parametrelerin değerleri.

\begin{tabular}{lllll}
\hline $\begin{array}{l}\text { Mn-0.15PIN- } \\
\text { O.55PMN- }\end{array}$ & $\mathrm{T}_{\mathrm{c}}$ & $\alpha\left(1 /{ }^{0} \mathrm{C}\right)$ & $-a_{4}$ & $a_{6}$ \\
0.30PT & & & & \\
\hline R-M & 112 & $1.908 \times 10^{-7}$ & $2.97 \times 10^{-3}$ & 1 \\
\hline
\end{tabular}

Denk. 9' un ikinci kısmı sadece $a_{4} / a_{6}$ oranını verdiği için $a_{6}$ katsayısı 1 olarak kabul edilmiştir. Şekil 1 de görüldüğü gibi, deneysel ters dielektrik katsayısının sıcaklık bağımlığı doğrusaldır ve doğrusal olan Denk. 9 bu deneysel verileri yeterli derecede karşılamaktadır $\left(R^{2}=0.998\right)$. Faz geçiş sıcaklığı sonrası (ferroelektrik-monoklinik faz) deneysel verilerin yetersizliğinden dolayı, bu fazda hesaplama yapılamamıştır. Çizelge 1 deki veriler kullanılarak, her iki Raman modu için sistemin kendiliğinden polarizasyonu hesaplanmıştır (Şekil 2). Bu çalışmada elektrik alanın sıfır olduğu durumda, kendiliğinden polarizasyon sistemin düzen parametresi olarak kabul edilmiştir. Rhombohedral-monoklinik faz geçişine yaklaştıkça sistemin düzeninde bozulma olması beklenir, yaptığımız hesaplamalar (Denk. 10) düzen parametresi olan kendiliğinden polarizasyonun sıcaklık artışı ile azaldığını göstermiştir (Şekil 2). Sistemde düzensizlik arttıkça, $510 \mathrm{~cm}^{-1}$ ve $584 \mathrm{~cm}^{-1}$ Raman modalarının frekansının sıcaklık artışı ile azaldığı, buna karşılık her iki Raman modu için çizgi genişliği (FWHM) değerinin arttığı gözlemlenmiştir (Liu et al. 2019).

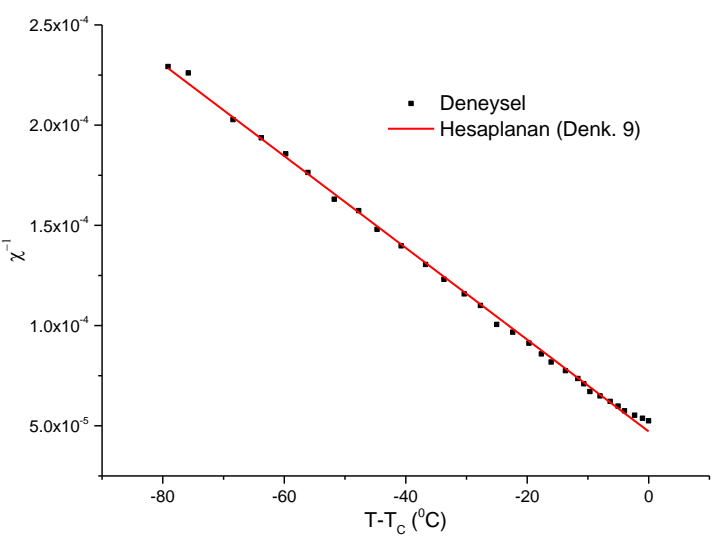

Şekil 1. Mn-0.15PIN-0.55PMN-0.30PT için ferroelektrikrhombohedral fazdaki $\left(T<T_{C}\right) \quad T-T_{C}$ 'nin bir fonksiyonu olarak Denk. (9)'dan hesaplanan ters duyarlılık $\chi^{-1}$, (口) deneysel verileri (Liu et al. 2019) temsil etmektedir.

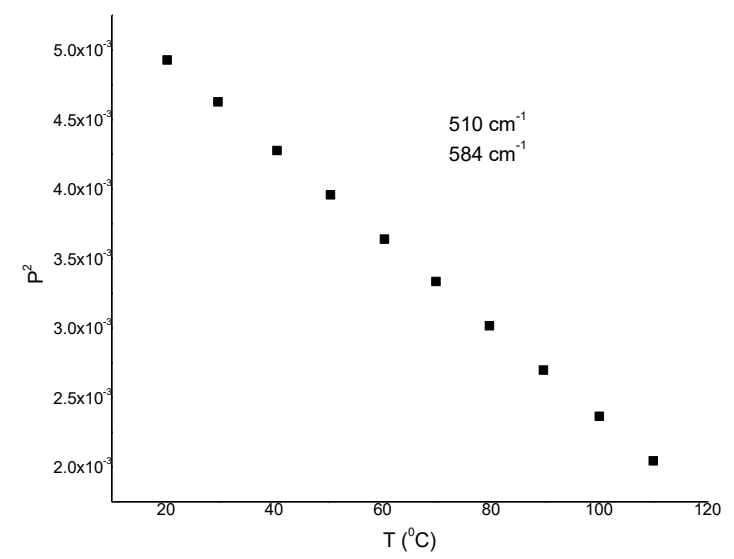

Şekil 2. Mn-0.15PIN-0.55PMN-0.30PT için T'nin bir fonksiyonu olarak Denk. (10)'dan hesaplanan kendiliğinden polarizasyon $\mathrm{P}^{2}\left(\mathrm{Tc}=112^{\circ} \mathrm{C}\right)$.

Raman modlarının bu çizgi genişliği artışı, sıcaklık artışına bağlı lokal simetrinin bozulmasından ve buna bağlı olarak da mikro yapısal bozukluktaki artıştan kaynaklanmaktadır. $\mathrm{Nb}-\mathrm{O}-\mathrm{Nb}$ gerilme modları olarak sınıflandırılan $510 \mathrm{~cm}^{-1}$ ve $584 \mathrm{~cm}^{-1}$ Raman modlarının sönümleme sabitinin (çizgi genişliği) sıcaklığa bağlılığı, Mn-0.15PIN-0.55PMN0.30PT için Şekil 3 ve 5'te çizildiği gibi psödospinfonon (Denk. 11) ve enerji dalgalanması (Denk. 12) modelleri kullanılarak hesaplanmıştır. Ferroelektrikrhombohedral faz için, sönümlenme sabitinin (çizgi genişliği) düzen parametresine $\left(P^{2}\right)$ ve sıcaklığa bağımlılığını tanımlamak için iki modeli (PS ve EF model) kullanarak, deneysel olarak ölçülen çizgi genişliği değerlerine iterasyon yaptık. Yaptığımız iterasyonlarda PS modelin Mn-0.15PIN-0.55PMN- 
0.30PT temel karışımının her iki Raman modu içinde daha iyi sonuç verdiği görülmüştür (Çizelge 2) (Şekil 3 ve 5$)$.

Çizelge 2. PS modeline (Denk. 11) ve EF modeline (Denk. 12) göre deneysel veriler (Liu et al. 2019) kullanılarak Raman modlarının (510 cm-1 ve $584 \mathrm{~cm}-1$ ) sönüm sabiti $\Gamma_{S P}$ için belirlenen katsayıların değerleri.

\begin{tabular}{lccccccc}
\hline & \multicolumn{3}{c}{ PS Model } & \multicolumn{5}{c}{ EF Model } \\
\hline $\begin{array}{l}\text { Raman } \\
\text { Mode }\end{array}$ & $\Gamma_{0}$ & $A_{0}$ & $\mathrm{R}^{2}$ & $\Gamma_{0}^{\prime}$ & $A_{0}^{\prime}$ & $\mathrm{R}^{2}$ & Sıcaklık Aralığı \\
\hline $\mathbf{5 1 0 \mathbf { ~ c m } ^ { - 1 }}$ & 102.76 & 3.32 & 0.83 & 103.35 & 2.06 & 0.71 & $20.1<\mathrm{T}<109.99$ \\
\hline $\mathbf{5 8 4} \mathbf{~ c m}^{-1}$ & 97.99 & 4.66 & 0.76 & 98.67 & 2.90 & 0.61 & $20.1<\mathrm{T}<109.99$ \\
\hline
\end{tabular}

Çizelge 3. PS ve EF modelleri kullanılarak belirtilen Raman modları için Denk. (14)'ten hesaplanan aktivasyon enerjisi değerleri.

\begin{tabular}{|c|c|c|c|c|c|}
\hline \multirow[t]{2}{*}{ Raman Mode } & \multicolumn{2}{|c|}{ PS Model } & \multicolumn{2}{|c|}{ EF Model } & \multirow[t]{2}{*}{ Sıcaklık Aralığı } \\
\hline & $\mathrm{U}(\mathrm{meV})$ & $\mathrm{C}$ & $\mathrm{U}(\mathrm{meV})$ & $\mathrm{C}$ & \\
\hline \multirow{3}{*}{$510 \mathrm{~cm}^{-1}$} & 3.12 & 4.76 & 2.18 & 4.73 & $20.21<T<40.52$ \\
\hline & 5.84 & 4.86 & 3.64 & 4.79 & $50.40<T<69.85$ \\
\hline & 16.76 & 5.22 & 13.83 & 5.12 & $79.73<T<100.03$ \\
\hline \multirow{3}{*}{$584 \mathrm{~cm}^{-1}$} & 4.56 & 4.77 & 3.18 & 4.73 & $20.21<T<40.52$ \\
\hline & 8.54 & 4.92 & 5.33 & 4.81 & $50.40<T<69.85$ \\
\hline & 24.00 & 5.43 & 19.86 & 5.29 & $79.73<T<100.03$ \\
\hline
\end{tabular}

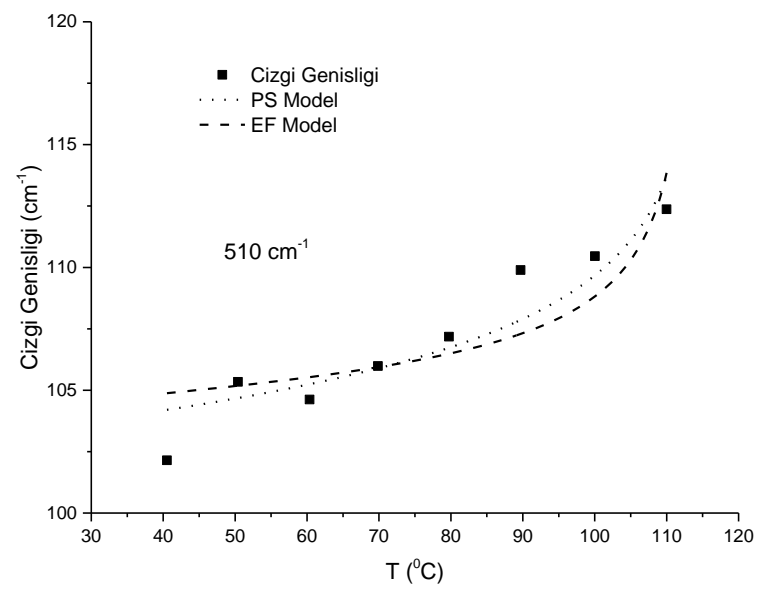

Şekil 3. Mn-0.15PIN-0.55PMN-0.30PT, $510 \mathrm{~cm}^{-1}$ Raman modu için, sıcaklığın bir fonksiyonu olarak sönümleme sabiti $\Gamma_{s p}$ (çizgi genişliği), ( $\left.\mathbf{a}\right)$ deneysel verileri (Liu et al. 2019) temsil etmektedir.

\section{Tartışma ve Sonuç}

Gevşeme (relaksiyon), pertürbe olmuş bir sistemin dengeye dönüşünü ifade etmektedir. Her bir gevşeme süreci, bir gevşeme zamanı $\tau$ ile kategorize edilir (Denk. 13). Dielektrik malzemelerde, kendiliğinden polarizasyon $(P)$ normalize edilmiş dış

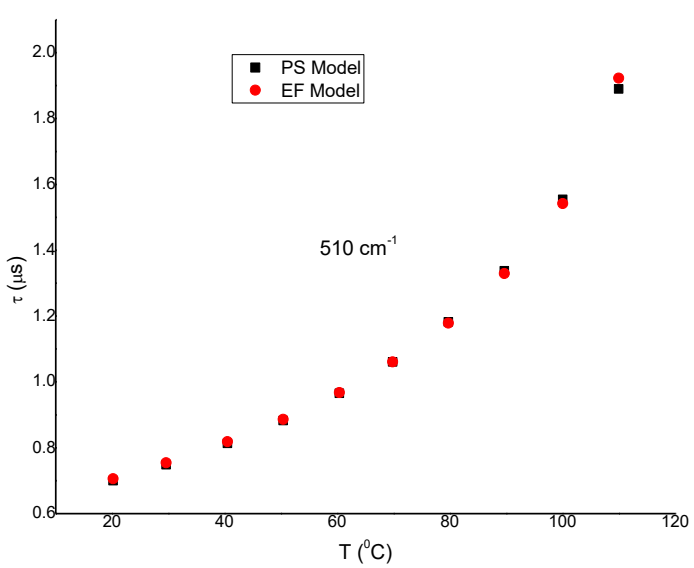

Şekil 4. Mn-0.15PIN-0.55PMN-0.30PT, $510 \mathrm{~cm}^{-1}$ Raman modu için, sıcaklığın bir fonksiyonu olarak gevşeme zamanı $(\tau)$

elektrik alanın büyüklüğüne (E) ve/veya ortam sıcaklığına bağlıdır. E ve/veya T değişirse, dielektrik polarizasyon yeni bir dengeye doğru gevşer. Yeni denge noktasına gelme süresi o malzemenin elektrik uygulamalarında son derece önemlidir. Yarı iletkenlerde, iletim işlemiyle nötrleştirilmenin ne kadar sürdüğünün bir ölçüsüdür. Gevşeme süresi 
metallerde küçük, yarı iletkenler ve izolatörlerde büyük değerlere sahiptir. Literatürdeki çalışmalarda farklı relaksör-ferroelektrik malzemeler için farkı frekans değerlerinde dielektrik katsayısının değişimleri incelenmiştir, bu ölçüler ile sistemin histerisis davranımı ve süreleri belirlenmiştir (Qian

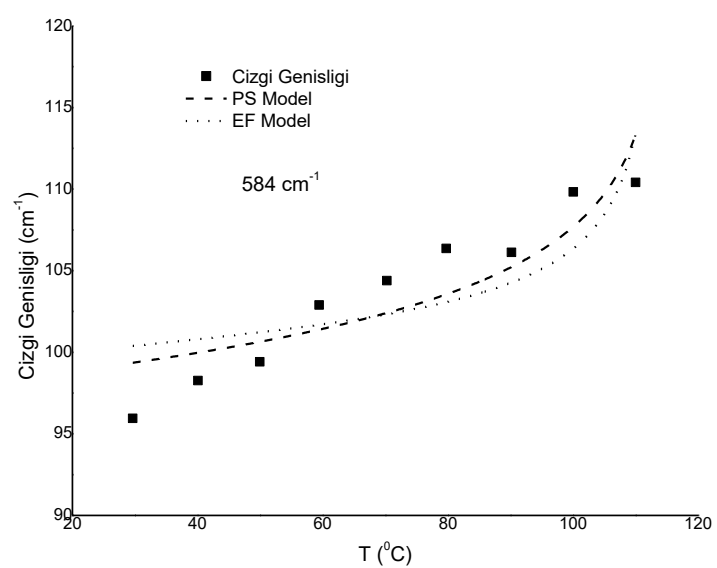

Şekil 5. Mn-0.15PIN-0.55PMN-0.30PT, $584 \mathrm{~cm}^{-1}$ Raman modu için, sıcaklığın bir fonksiyonu olarak sönümleme sabiti $\Gamma_{s p}$ (çizgi genişliği), ( $\left.\mathbf{\square}\right)$ deneysel verileri (Liu et al. 2019) temsil etmektedir.

Bu çalışmada, Mn-0.15PIN-0.55PMN-0.30PT kristal malzemenin her iki Raman modu için gevşeme zamanının sıcaklığa bağlıı̆ı, PS ve EF model için hesaplanan sönümleme sabiti değerleri kullanılarak hesaplanmıştır. Ferroelektrik-rhombohedral fazda, her iki mod içinde sıcaklık artışı ile gevşeme süresinin arttığı, faz geçiş noktasında $\left(T_{c}\right)$ süreksiz olduğu görülmüştür (Şekil 4 ve 6). Mn-0.15PIN0.55PMN-0.30PT kristal malzemesinin gevşeme süresindeki artış diğer ferroelektrik özellik gösteren malzemeler kıyaslandığında daha az değişmektedir (Qian et al., 2013, Sun et al. 2016, Cowley at al. 2011). Sıcaklığa bağlı gevşeme süresindeki bu kademeli artış, Mn-0.15PIN-0.55PMN-0.30PT kristal malzemesinin sıcaklık artışı ile yarı iletken özelliklerinin arttığını göstermektedir. Son yıllardaki çalışmalar bu malzemenin rhombohedralmonoklinik faz geçiş bölgesindeki davranımı sayesinde yüksek sığaya sahip kapasitörlerde dielektrik malzeme olarak kullanılabileceğini, böylelikle enerji depolama için verimli bir malzeme olacağını göstermektedir (Cowley et al. 2011). Bu et al., 2013, Sun et al. 2016, Cowley at al. 2011). Ancak, relaksör-ferroelektrik malzemelerin gevşeme süresi davranımının doğruluğu, halihazırda ileri teorik ve deneysel çalışmalara ihtiyaç duymaktadır (Zhang and Huang 2020).

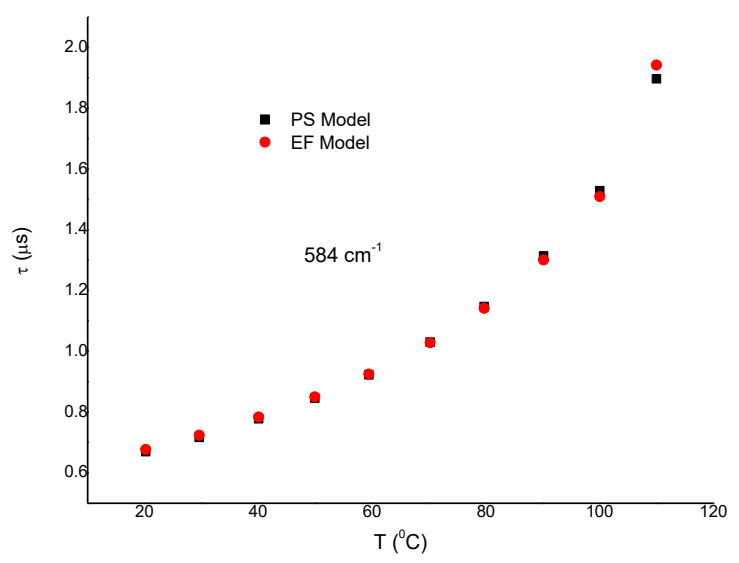

Şekil 6. Mn-0.15PIN-0.55PMN-0.30PT, $584 \mathrm{~cm}^{-1}$ Raman modu için, sıcaklığın bir fonksiyonu olarak gevşeme zamanı $(\tau)$

çalışmada son olarak, Mn-0.15PIN-0.55PMN-0.30PT kristal malzemesinin, her iki Raman modunda PS ve EF modelleri için hesaplanan çizgi genişliği (sönümleme sabiti) değerleri kullanılarak, rhombohedral fazda farklı sıcaklık aralıkları için aktivasyon enerjileri hesaplanmıştır (Çizelge 3). Bu malzeme için literatürde kıyaslama yapılabilecek aktivasyon enerjisi değeri bulunmamasına rağmen, bulduğumuz aktivasyon enerjisi değeri literatürde bulunan faz geçiş sıcaklığına yakın sıcaklık bölgesi için farklı konsantrasyondaki benzer malzeme olan $\mathrm{Pb}\left(\operatorname{In}_{1 / 2} \mathrm{Nb}_{1 / 2}\right) \mathrm{O}_{3}-\mathrm{Pb}\left(\mathrm{Mg}_{1 / 3} \mathrm{Nb}_{2 / 3}\right) \mathrm{O}_{3}-\mathrm{PbTiO}_{3} \quad$ (PINPMN-PT) için sunulan aktivasyon enerjisi ile uyumlu çıkmıştır (Sun et al. 2016). Her iki model ve Raman modu için faz geçiş sıcaklığına yaklaştıkça aktivasyon enerjisinin beklendiği gibi arttığı görülmüştür. Aktivasyon enerjisindeki bu artışın sebebi, lokal simetrinin bozulması ve buna bağlı moleküler fonon frekanslarının artmasından kaynaklandığı değerlendirilmiştir.

Bu çalışmada, Mn katkılı 0.15PIN-0.55PMN-0.30PT tekil kristalinin, ferroelektrik-rhombohedral fazda 
dielektrik duyarlık sabitinin, kendiliğinden polarizasyonun ve gevşeme zamanının sıcaklık bağımlılığını Landau Fenomolojik ortalama alan modelinden elde ettik. Dielektrik duyarlık sabiti için türetilen bağıntımızı, Mn-0.15PIN-0.55PMN-0.30PT tekil kristal için literatürdeki deneysel verilere fit ederek ortalama alan serbest enerjisinde verilen katsayıları belirledik. Bu katsayıları kullanarak kendiliğinden polarizasyonu sıcaklığa bağlı olarak hesapladık. Literatürdeki deneysel sönümlenme sabiti (çizgi genişliği) verilerine, PS ve EF model için türetilen denklemlere fit yaptık. Deneysel verilerle karşılaştırıldığında, PS modelinden hesaplanan sönümleme sabiti, EF modelinden hesaplanandan daha iyi sonuç vermiştir. Hesaplanan polarizasyon ve sönümleme sabiti değerlerini kullanarak, her iki model için gevşeme zamanını hesapladık. Gevşeme zamanının sıcaklık arttıkça üstel olarak arttığı görülmüştür. Son olarak, $510 \mathrm{~cm}^{-1}$ ve $584 \mathrm{~cm}^{-1}$ Raman modları için aktivasyon enerjilerini hesapladık, beklendiği gibi aktivasyon enerjisinin faz geçiş sıcaklığına yaklaştıkça arttığı görülmüştür.

\section{Kaynaklar}

Blinc, R., Dolinšek, J., Gregorovič, A., Zalar, B., Filipič, C., Kutnjak, Z., Levstic, A. and Pirc, R., 1999. Local Polarization Distribution and Edwards-Anderson Order Parameter of Relaxor Ferroelectrics. Physical Review Letters, 83(2), 424-427.

Böttger, U., Biermann, A. and Arlt, G., 1992. PtP240. Phase transitions in PLZT ceramics. Ferroelectrics, 134(1), 253-258.

Burns, G. and Dacol, F.H., 1983. Glassy polarization behavior in ferroelectric compounds $\mathrm{Pb}\left(\mathrm{Mg}_{1 / 3} \mathrm{Nb}_{2 / 3}\right) \mathrm{O}_{3}$ and $\mathrm{Pb}\left(\mathrm{Zn}_{1 / 3} \mathrm{Nb}_{2 / 3}\right) \mathrm{O}_{3}$. Solid State Communications, 48(10), 853-856.

Colla, E.V., Koroleva, E.Y., Okuneva, N.M. and Vakhrushev, S.B., 1995. Long-Time Relaxation of the Dielectric Response in Lead Magnoniobate. Physical Review Letters, 74(9), 1681-1684.

Cowley, R.A., Gvasaliya, S.N., Lushnikov, S.G., Roessli, B. and Rotaru, G.M., 2011. Relaxing with relaxors: a review of relaxor ferroelectrics. Advances in Physics, 60(2), 229-327.

Cross, L.E., 1987. Relaxor ferroelectrics. Ferroelectrics, 76(1), 241-267.
Cross, L.E., 1993. Ferroelectric Ceramics: Tailoring Properties for Specific Applications, Paper presented at the Ferroelectric Ceramics, Basel.

Davis, M., Damjanovic, D. and Setter, N., 2005. Electricfield-induced orthorhombic to rhombohedral phase transition in [111]C-oriented $0.92 \mathrm{~Pb}\left(\mathrm{Zn}_{1 / 3} \mathrm{Nb}_{2 / 3}\right) \mathrm{O}_{3}-0.08 \mathrm{PbTiO}_{3}$. Journal of Applied Physics, 97(6), 064101.

Davis, M., Damjanovic, D. and Setter, N., 2006. Electricfield-, temperature-, and stress-induced phase transitions in relaxor ferroelectric single crystals. Physical Review B, 73(1), 014115.

Hendrickson, J.R. and Bray, P.J., 1973. A phenomenological equation for NMR motional narrowing in solids. Journal of Magnetic Resonance, 9(3), 341-357.

Kim, I., Jang, K., Kim, I. and Li, L., 2018. Higher-order Landau phenomenological models for perovskite crystals based on the theory of singularities: a new phenomenology of $\mathrm{BaTiO}_{3}$. Phase Transitions, 91(3), 239-253.

Kurt, M., Yurtseven, H., Kurt, A. and Aksoy, S., 2019. Calculation of the infrared frequency and the damping constant (full width at half maximum) for metal organic frameworks. Chinese Physics B, 28(6), 066401.

Kutnjak, Z., Garland, C.W., Schatz, C.G., Collings, P.J., Booth, C.J. and Goodby, J.W., 1996. Critical point for the blue-phase-III--isotropic phase transition in chiral liquid crystals. Physical Review E, 53(5), 4955-4963.

Kutnjak, Z., Petzelt, J. and Blinc, R., 2006. The giant electromechanical response in ferroelectric relaxors as a critical phenomenon. Nature, 441(7096), 956959.

Lahajnar, G., Blinc, R. and Zumer, S., 1974. Proton spinlattice relaxation by critical polarization fluctuations in $\mathrm{KH}_{2} \mathrm{PO}_{4}$. Physics of condensed matter, 18(4), 301316.

Liu, F., Chen, J., Zhu, R., Zhao, J., Xue, S., Du, Q., Wang, F. and Luo, H., 2019. Temperature Dependence of Electrical Properties and Phase Transition Characteristics of [001]-Oriented Rhombohedral Mn0.15PIN-0.55PMN-0.30PT Single Crystal. Physica Status Solidi (a), 216(23), 1900457-1 - 1900457-6.

Liu, X., Fang, B., Deng, J., Yan, H., Deng, H., Yue, Q., Ding, J., Zhano, X. and Luo, H., 2016. Study of temperature- 
dependent Raman spectroscopy and electrical properties in [001]-oriented $0.35 \mathrm{~Pb}\left(\ln _{1 / 2} \mathrm{Nb}_{1 / 2}\right) \mathrm{O}_{3}$ $0.35 \mathrm{~Pb}\left(\mathrm{Mg}_{1 / 3} \mathrm{Nb}_{2 / 3}\right) \mathrm{O}_{3}-0.30 \mathrm{PbTiO}_{3}-\mathrm{Mn}$ single crystals. Journal of Applied Physics, 119(1), 014105-1 014105-6.

Lu, X., Fan, J., Zhang, H., Wu, H., Li, H., Cao, W., 2020. Phase stability and Landau phenomenological model of relaxor ferroelectric single crystals 0.78Pb(Mg1/3Nb2/3)O3-0.22PbTiO3, Ceramics International, (DOI: 10.1016/j.ceramint.2020.12.125).

Matsushita, M., 1976. Anomalous temperature dependence of the frequency and damping constant of phonons near $T \lambda$ in ammonium halides. The Journal of Chemical Physics, 65(1), 23-28.

Pirc, R., Blinc, R. and Kutnjak, Z., 2002. Nonlinear dielectric response of relaxor ferroelectrics. Physical Review B, 65(21), 214101-1 - 214101-7.

Qian, K., Fang, B., Du, Q., Ding, J., Zhao, X. and Luo, H., 2013. Phase development and electrical properties of $\mathrm{Pb}\left(\mathrm{Mg}_{1 / 3} \mathrm{Nb}_{2 / 3}\right) \mathrm{O}_{3}-\mathrm{PbTiO}_{3}$ ceramics prepared by partial oxalate route. Physica Status Solidi (a), 210(6), 11491156.

Schaack, G. and Winterfeldt, V., 1977. Temperature behaviour of optical phonons near Tc in triglycine sulphate and triglycine selenate. Ferroelectrics, 15(1), 35-41.

Sun, E., Qi, X., Yuan, Z., Sang, S., Zhang, R., Yang, B., Cao, W. and Zhao, L., 2016. Relaxation behavior in $0.24 \mathrm{~Pb}\left(\mathrm{In}_{1 / 2} \mathrm{Nb}_{1 / 2}\right) \mathrm{O}_{3}-0.49 \mathrm{~Pb}\left(\mathrm{Mg}_{1 / 3} \mathrm{Nb}_{2 / 3}\right) \mathrm{O}_{3}-$ $0.27 \mathrm{PbTiO}_{3}$ ferroelectric single crystal. Ceramics International, 42(4), 4893-4898.

Svitelskiy, O., Toulouse, J., Yong, G. and Ye, Z.G., 2003. Polarized Raman study of the phonon dynamics in
$\mathrm{PbMg}_{1 / 3} \mathrm{Nb}_{2 / 3} \mathrm{O}_{3}$ crystal. Physical Review B, 68(10), 104107-1 - 104107-10.

Viehland, D. and Powers, J., 2001. Effect of uniaxial stress on the electromechanical properties of $0.7 \mathrm{~Pb}\left(\mathrm{Mg}_{1 / 3} \mathrm{Nb}_{2 / 3}\right) \mathrm{O}_{3}-0.3 \mathrm{PbTiO}_{3}$ crystals and ceramics. Journal of Applied Physics, 89(3), 1820-1825.

Wang, Y., Sun, E., Song, W., Li, W., Zhang, R. and Cao, W., 2014. Improved thermal stability of [001]c poled $0.24 \mathrm{~Pb}\left(\mathrm{In}_{1 / 2} \mathrm{Nb}_{1 / 2}\right) \mathrm{O}_{3}-0.47 \mathrm{~Pb}\left(\mathrm{Mg}_{1 / 3} \mathrm{Nb}_{2 / 3}\right) \mathrm{O}_{3}-$ $0.29 \mathrm{PbTiO}_{3}$ single crystal with manganese doping. Journal of Alloys and Compounds, 601, 154-157.

Westphal, V., Kleemann, W. and Glinchuk, M.D., 1992. Diffuse phase transitions and random-field-induced domain states of the "relaxor" ferroelectric $\mathrm{PbMg}_{1 / 3} \mathrm{Nb}_{2 / 3} \mathrm{O}_{3}$. Physical Review Letters, 68(6), 847 850.

Yamada, Y., Mori, M. and Noda, Y., 1972. A Microscopic Theory on the Phase Transitions in $\mathrm{NH}_{4} \mathrm{Br}$; An Ising Spin Phonon Coupled System. Journal of the Physical Society of Japan, 32(6), 1565-1576.

Yurtseven, H., Celik, M. and Karacali, H., 2017. Calculation of the Spontaneous Polarization and the Dielectric Constant for the Ferroelectric $\mathrm{N}\left(\mathrm{CH}_{3}\right)_{4} \mathrm{HSO}_{4}$ Using the Mean Field Model. High Temperature Materials and Processes, 36(9), 863-869.

Zhang, L.L. and Huang, Y.N., 2020. Theory of relaxorferroelectricity. Scientific Reports, 10(1), 5060-5077.

Zhao, X., Qu, W., Tan, X., Bokov, A.A. and Ye, Z.G., 2007. Electric field-induced phase transitions in (111)-, (110)- and (100)-oriented $\mathrm{Pb}\left(\mathrm{Mg}_{1 / 3} \mathrm{Nb}_{2 / 3}\right) \mathrm{O}_{3}$ single crystals. Physical Review B, 75(10), 104106-1 104106-12. 